\title{
A Tableau Algorithm for Fuzzy Description Logics over Residuated De Morgan Lattices
}

\author{
Stefan Borgwardt and Rafael Peñaloza \\ Theoretical Computer Science, TU Dresden, Germany \\ \{stefborg, penaloza\}@tcs.inf.tu-dresden.de
}

\begin{abstract}
Fuzzy description logics can be used to model vague knowledge in application domains. This paper analyses the consistency and satisfiability problems in the description logic $\mathcal{S H I}$ with semantics based on a complete residuated De Morgan lattice. The problems are undecidable in the general case, but can be decided by a tableau algorithm when restricted to finite lattices. For some sublogics of $\mathcal{S H \mathcal { I }}$, we provide upper complexity bounds that match the complexity of crisp reasoning.
\end{abstract}

\section{Introduction}

Description Logics (DLs) [1] are a family of knowledge representation formalisms that are widely used to model application domains. In DLs, knowledge is represented with the help of concepts (unary predicates) and roles (binary predicates) that express the relationships between concepts. They have been successfully employed to formulate ontologies-especially in the medical domain-like Galen ${ }^{1}$ and serve as the underpinning for the current semantic web language OWL $2{ }^{2}$ Standard reasoning in these logics includes concept satisfiability (is a given concept non-contradictory?) and ontology consistency (does a given ontology have a model?). These and other reasoning problems have been studied for DLs, and several algorithms have been proposed and implemented.

One of the main challenges in knowledge representation is the correct modeling and use of imprecise or vague knowledge. For example, medical diagnoses from experts are rarely clear-cut and usually depend on concepts like HighBloodPressure that are necessarily vague. Fuzzy variants of description logics were introduced in the nineties as a means to tackle this challenge. Their applicability to the representation of medical knowledge was studied in [22].

Fuzzy DLs generalize (crisp) DLs by providing a membership degree semantics for their concepts. Thus, e.g. 130/85 belongs to the concept HighBloodPressure with a lower degree than, say 140/80. In their original form, membership degrees are elements of the real-number interval $[0,1]$, but this was later generalized to lattices $[21,26]$. The papers $[21,26]$ consider only a limited kind of semantics over lattices, where conjunction and disjunction are interpreted through the lattice operators meet and join, respectively.

\footnotetext{
${ }^{1}$ http://www.opengalen.org/

${ }^{2}$ http://www.w3.org/TR/owl2-overview/
} 
In this paper, we consider a more general lattice-based semantics that uses a triangular norm (t-norm) and its residuum as interpretation functions for the logical constructors. We study fuzzy variants of the standard reasoning problems like concept satisfiability and ontology consistency in this setting.

We show that concept satisfiability in $\mathcal{A L C}$ under this semantics is undecidable in general, even if we restrict ourselves to a very simple class of infinite lattices. However, we show with the help of a tableaux-based algorithm that decidability of reasoning can be regained - even for the more expressive DL $\mathcal{S H I}$ - if the underlying lattice is required to be finite. Moreover, we describe a black-box method that can be used to transform any decision algorithm for (a small generalization of) satisfiability into a decision procedure for consistency.

Due to space constraints, some of the technical proofs have been left out of this paper; they can be found in the technical report [12].

\section{Preliminaries}

We start with a short introduction to residuated lattices, which will be the base for the semantics of the fuzzy DL $L-\mathcal{S H I}$. For a more comprehensive view on these lattices, we refer the reader to $[15,17]$.

\section{$2.1 \quad$ Lattices}

A lattice is a triple $(L, \vee, \wedge)$, consisting of a carrier set $L$ and two idempotent, associative, and commutative binary operators join $\vee$ and meet $\wedge$ on $L$ that satisfy the absorption laws $\ell_{1} \vee\left(\ell_{1} \wedge \ell_{2}\right)=\ell_{1}=\ell_{1} \wedge\left(\ell_{1} \vee \ell_{2}\right)$ for all $\ell_{1}, \ell_{2} \in L$. These operations induce a partial order $\leq$ on $L: \ell_{1} \leq \ell_{2}$ iff $\ell_{1} \wedge \ell_{2}=\ell_{1}$. As usual, we write $\ell_{1}<\ell_{2}$ if $\ell_{1} \leq \ell_{2}$ and $\ell_{1} \neq \ell_{2}$. A subset $T \subseteq L$ is called an antichain (in $L$ ) if there are no two elements $\ell_{1}, \ell_{2} \in T$ with $\ell_{1}<\ell_{2}$. Whenever it is clear from the context, we will use the carrier set $L$ to represent the lattice $(L, \vee, \wedge)$.

The lattice $L$ is distributive if $\vee$ and $\wedge$ distribute over each other, finite if $L$ is finite, and bounded if it has a minimum and a maximum element, denoted as $\mathbf{0}$ and $\mathbf{1}$, respectively. It is complete if joins and meets of arbitrary subsets $T \subseteq L, \bigvee_{t \in T} t$ and $\bigwedge_{t \in T} t$, respectively, exist. Clearly, every finite lattice is also complete, and every complete lattice is bounded.

A De Morgan lattice is a bounded distributive lattice $L$ extended with an involutive and anti-monotonic unary operation $\sim$, called (De Morgan) negation, satisfying the De Morgan laws $\sim\left(\ell_{1} \vee \ell_{2}\right)=\sim \ell_{1} \wedge \sim \ell_{2}$ and $\sim\left(\ell_{1} \wedge \ell_{2}\right)=\sim \ell_{1} \vee \sim \ell_{2}$ for all $\ell_{1}, \ell_{2} \in L$.

Given a lattice $L$, a $t$-norm is an associative and commutative binary operator on $L$ that is monotonic and has $\mathbf{1}$ as its unit. A residuated lattice is a lattice $L$ with a t-norm $\otimes$ and a binary operator $\Rightarrow$ (called residuum) such that for all $\ell_{1}, \ell_{2}, \ell_{3} \in L$ we have $\ell_{1} \otimes \ell_{2} \leq \ell_{3}$ iff $\ell_{2} \leq \ell_{1} \Rightarrow \ell_{3}$. A simple consequence is that for all $\ell_{1}, \ell_{2} \in L$ we have $\mathbf{1} \Rightarrow \ell_{1}=\ell_{1}$, and $\ell_{1} \leq \ell_{2}$ iff $\ell_{1} \Rightarrow \ell_{2}=\mathbf{1}$.

A t-norm $\otimes$ over a complete lattice $L$ is continuous if for all $\ell \in L$ and $T \subseteq L$ we have $\ell \otimes\left(\bigvee_{\ell^{\prime} \in T} \ell^{\prime}\right)=\bigvee_{\ell^{\prime} \in T}\left(\ell \otimes \ell^{\prime}\right)$. Every continuous t-norm $\otimes$ has the unique 


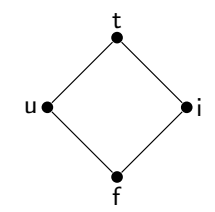

Fig. 1. The De Morgan residuated lattice $L_{4}$ with $\sim \mathrm{u}=\mathrm{u}$ and $\sim \mathrm{i}=\mathrm{i}$.

residuum $\Rightarrow$ defined by $\ell_{1} \Rightarrow \ell_{2}=\bigvee\left\{x \mid \ell_{1} \otimes x \leq \ell_{2}\right\}$ for all $\ell_{1}, \ell_{2} \in L$. If $L$ is a distributive lattice, then the meet operator $\ell_{1} \wedge \ell_{2}$ always defines a continuous t-norm, often called the Gödel t-norm. In a residuated De Morgan lattice $L$, the t-conorm $\oplus$ is defined as as $\ell_{1} \oplus \ell_{2}:=\sim\left(\sim \ell_{1} \otimes \sim \ell_{2}\right)$. The $t$-conorm of the Gödel t-norm is the join operator $\ell_{1} \vee \ell_{2}$.

For example, consider the finite lattice $L_{4}$, with the elements $\mathrm{f}, \mathrm{u}, \mathrm{i}$, and $\mathrm{t}$ as shown in Figure 1. This lattice has been used for reasoning about incomplete and contradictory knowledge [5] and as a basis for a paraconsistent rough DL [28]. In our blood pressure scenario, the two degrees i and u may be used to express readings that are potentially and partially high blood pressures, respectively. The incomparability of these degrees reflects the fact that none of them can be stated to belong more to the concept HighBloodPressure than the other.

For the rest of this paper, $L$ denotes a complete residuated De Morgan lattice with t-norm $\otimes$ and residuum $\Rightarrow$, unless explicitely stated otherwise.

\subsection{The Fuzzy DL $L-\mathcal{S H} \mathcal{I}$}

The fuzzy DL $L-\mathcal{S H I}$ is a generalization of the crisp DL $\mathcal{S H \mathcal { I }}$ that uses the elements of $L$ as truth values, instead of just the Boolean true and false. The syntax of $L-\mathcal{S H I}$ is the same as in $\mathcal{S H I}$ with the addition of the constructor $\rightarrow$.

Definition 1 (syntax of $L-\mathcal{S H} \mathcal{I}$ ). Let $\mathrm{N}_{\mathrm{C}}, \mathrm{N}_{\mathrm{R}}$, and $\mathrm{N}_{\mathrm{I}}$ be pairwise disjoint sets of concept-, role-, and individual names, respectively, and $\mathrm{N}_{\mathrm{R}}^{+} \subseteq \mathrm{N}_{\mathrm{R}}$ a set of transitive role names. The set of (complex) roles is $\mathrm{N}_{\mathrm{R}} \cup\left\{r^{-} \mid r \in \mathrm{N}_{\mathrm{R}}\right\}$. The set of (complex) concepts $C$ is obtained through the following syntactic rule, where $A \in \mathrm{N}_{\mathrm{C}}$ and $s$ is a complex role:

$$
C::=A\left|C_{1} \sqcap C_{2}\right| C_{1} \sqcup C_{2}\left|C_{1} \rightarrow C_{2}\right| \neg C|\exists s . C| \forall s . C|\top| \perp .
$$

The inverse of a complex role $s$ (denoted by $\bar{s}$ ) is $s^{-}$if $s \in \mathrm{N}_{\mathrm{R}}$ and $r$ if $s=r^{-}$. A complex role $s$ is transitive if either $s$ or $\bar{s}$ belongs to $\mathrm{N}_{\mathrm{R}}^{+}$.

The semantics of this logic is based on functions specifying the membership degree of every domain element in a concept $C$.

Definition 2 (semantics of $L-\mathcal{S H} \mathcal{I})$. An interpretation is a pair $\mathcal{I}=\left(\Delta^{\mathcal{I}},{ }^{\mathcal{I}}\right.$ ) where $\Delta^{\mathcal{I}}$ is a non-empty domain, and ${ }^{\mathcal{I}}$ is a function that assigns to every individual name a an element $a^{\mathcal{I}} \in \Delta^{\mathcal{I}}$, to every concept name $A$ a function 
$A^{\mathcal{I}}: \Delta^{\mathcal{I}} \rightarrow L$, and to every role name $r$ a function $r^{\mathcal{I}}: \Delta^{\mathcal{I}} \times \Delta^{\mathcal{I}} \rightarrow L$, where $r^{\mathcal{I}}(x, y) \otimes r^{\mathcal{I}}(y, z) \leq r^{\mathcal{I}}(x, z)$ holds for all $r \in \mathrm{N}_{\mathrm{R}}^{+}$and $x, y, z \in \Delta^{\mathcal{I}}$.

The function. ${ }^{\mathcal{I}}$ is extended to $L-\mathcal{S H I}$ concepts as follows for every $x \in \Delta^{\mathcal{I}}$ :

$-\top^{\mathcal{I}}(x)=\mathbf{1}, \quad \perp^{\mathcal{I}}(x)=\mathbf{0}$,

$-(C \sqcap D)^{\mathcal{I}}(x)=C^{\mathcal{I}}(x) \otimes D^{\mathcal{I}}(x), \quad(C \sqcup D)^{\mathcal{I}}(x)=C^{\mathcal{I}}(x) \oplus D^{\mathcal{I}}(x)$,

$-(C \rightarrow D)^{\mathcal{I}}(x)=C^{\mathcal{I}}(x) \Rightarrow D^{\mathcal{I}}(x)$,

$-(\neg C)^{\mathcal{I}}(x)=\sim C^{\mathcal{I}}(x)$,

- $(\exists s . C)^{\mathcal{I}}(x)=\bigvee_{y \in \Delta^{\mathcal{I}}}\left(s^{\mathcal{I}}(x, y) \otimes C^{\mathcal{I}}(y)\right)$,

- $(\forall s . C)^{\mathcal{I}}(x)=\bigwedge_{y \in \Delta^{\mathcal{I}}}\left(s^{\mathcal{I}}(x, y) \Rightarrow C^{\mathcal{I}}(y)\right)$,

where $\left(r^{-}\right)^{\mathcal{I}}(x, y)=r^{\mathcal{I}}(y, x)$ for all $x, y \in \Delta^{\mathcal{I}}$ and $r \in \mathrm{N}_{\mathrm{R}}$.

The semantics of the existential and value restrictions is just the direct application of the semantics of quantification of fuzzy first-order logic $[18,19]$ to fuzzy DLs.

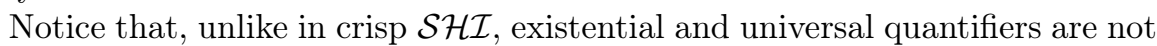
dual to each other, i.e. in general, $(\neg \exists s . C)^{\mathcal{I}}(x)=(\forall s . \neg C)^{\mathcal{I}}(x)$ does not hold. Likewise, the implication constructor $\rightarrow$ cannot be expressed in terms of the negation $\neg$ and conjunction $\sqcap$.

The axioms of this logic are those of crisp $\mathcal{S H I}$, but with associated lattice values, which express the degree to which the restrictions must be satisfied.

Definition 3 (axioms). An assertion can be a concept assertion of the form $\langle a: C \triangleright \ell\rangle$ or a role assertion of the form $\langle(a, b): s \triangleright \ell\rangle$, where $C$ is a concept, $s$ is a complex role, $a, b$ are individual names, $\ell \in L$, and $\triangleright \in\{=, \geq\}$. If $\triangleright$ is $=$, then it is called an equality assertion. A general concept inclusion (GCI) is of the form $\langle C \sqsubseteq D, \ell\rangle$, where $C, D$ are concepts, and $\ell \in L$. $A$ role inclusion is of the form $s \sqsubseteq s^{\prime}$, where $s$ and $s^{\prime}$ are complex roles.

An ontology $(\mathcal{A}, \mathcal{T}, \mathcal{R})$ consists of a finite set $\mathcal{A}$ of assertions (ABox), a finite set $\mathcal{T}$ of GCIs (TBox), and a finite set $\mathcal{R}$ of role inclusions (RBox). The $A B o x \mathcal{A}$ is called local if there is an individual $a \in N_{\mathrm{I}}$ such that all assertions in $\mathcal{A}$ are of the form $\langle a: C=\ell\rangle$, for some concept $C$ and $\ell \in L$.

An interpretation $\mathcal{I}$ satisfies the assertion $\langle a: C \triangleright \ell\rangle$ if $C^{\mathcal{I}}\left(a^{\mathcal{I}}\right) \triangleright \ell$ and the assertion $\langle(a, b): s \triangleright \ell\rangle$ if $s^{\mathcal{I}}\left(a^{\mathcal{I}}, b^{\mathcal{I}}\right) \triangleright \ell$. It satisfies the $G C I\langle C \sqsubseteq D, \ell\rangle$ if $C^{\mathcal{I}}(x) \Rightarrow D^{\mathcal{I}}(x) \geq \ell$ holds for every $x \in \Delta^{\mathcal{I}}$. It satisfies the role inclusion $s \sqsubseteq s^{\prime}$ if for all $x, y \in \Delta^{\mathcal{I}}$ we have $s^{\mathcal{I}}(x, y) \leq s^{\mathcal{I}}(x, y)$.

$\mathcal{I}$ is a model of the ontology $(\mathcal{A}, \mathcal{T}, \mathcal{R})$ if it satisfies all axioms in $\mathcal{A}, \mathcal{T}, \mathcal{R}$.

Given an RBox $\mathcal{R}$, the role hierarchy $\sqsubseteq \mathcal{R}$ on the set of complex roles is the reflexive and transitive closure of the relation

$$
\left\{\left(s, s^{\prime}\right) \mid s \sqsubseteq s^{\prime} \in \mathcal{R} \text { or } \bar{s} \sqsubseteq \overline{s^{\prime}} \in \mathcal{R}\right\} .
$$

Using reachability algorithms, the role hierarchy can be computed in polynomial time in the size of $\mathcal{R}$. An RBox $\mathcal{R}$ is called acyclic if it contains no cycles of the form $s \sqsubseteq_{\mathcal{R}} s^{\prime}, s^{\prime} \sqsubseteq_{\mathcal{R}} s$ for two roles $s \neq s^{\prime}$. 
The fuzzy DL $L-\mathcal{A L C}$ is the sublogic of $L-\mathcal{S H I}$ where no role inclusions, transitive roles, or inverse roles are allowed. $\mathcal{S H I}$ is the sublogic of $L-\mathcal{S H I}$ where the underlying lattice contains only the elements $\mathbf{0}$ and $\mathbf{1}$, which may be interpreted as false and true, respectively, and the t-norm and t-conorm are conjunction and disjunction, respectively.

Recall that the semantics of the quantifiers require the computation of a supremum or infimum of the membership degrees of a possibly infinite set of elements of the domain. To obtain effective decision procedures, reasoning is usually restricted to a special kind of models, called witnessed models [19].

Definition 4 (witnessed model). Let $n \in \mathbb{N}$. A model $\mathcal{I}$ of an ontology $\mathcal{O}$ is $n$-witnessed if for every $x \in \Delta^{\mathcal{I}}$, every role $s$ and every concept $C$ there are $x_{1}, \ldots, x_{n}, y_{1}, \ldots, y_{n} \in \Delta^{\mathcal{I}}$ such that

$$
(\exists s . C)^{\mathcal{I}}(x)=\bigvee_{i=1}^{n}\left(s^{\mathcal{I}}\left(x, x_{i}\right) \otimes C^{\mathcal{I}}\left(x_{i}\right)\right), \quad(\forall s . C)^{\mathcal{I}}(x)=\bigwedge_{i=1}^{n}\left(s^{\mathcal{I}}\left(x, y_{i}\right) \Rightarrow C^{\mathcal{I}}\left(y_{i}\right)\right) .
$$

In particular, if $n=1$, the suprema and infima from the semantics of $\exists$ s.C and $\forall s . C$ are maxima and minima, respectively, and we say that $\mathcal{I}$ is witnessed.

The reasoning problems for $\mathcal{S H I}$ generalize to the fuzzy semantics of $L-\mathcal{S H I}$.

Definition 5 (decision problems). Let $\mathcal{O}$ be an ontology, $C, D$ be two concepts, $a \in \mathrm{N}_{\mathrm{l}}$, and $\ell \in L . \mathcal{O}$ is consistent if it has a (witnessed) model. $C$ is strongly $\ell$-satisfiable if there is a (witnessed) model $\mathcal{I}$ of $\mathcal{O}$ and $x \in \Delta^{\mathcal{I}}$ with $C^{\mathcal{I}}(x) \geq \ell$. The individual $a$ is an $\ell$-instance of $C$ if $\langle a: C \geq \ell\rangle$ is satisfied by all (witnessed) models of $\mathcal{O} . C$ is $\ell$-subsumed by $D$ if $\langle C \sqsubseteq D, \ell\rangle$ is satisfied by all (witnessed) models of $\mathcal{O}$.

Example 6. It is known that coffee drinkers and salt consumers tend to have a higher blood pressure. On the other hand, bradycardia is highly correlated with a lower blood pressure. This knowledge can be expressed through the TBox

$\{\langle$ CoffeeDrinker $\sqsubseteq$ HighBloodPressure, i $\rangle,\langle$ SaltConsumer $\sqsubseteq$ HighBloodPressure, i $\rangle$,

$$
\langle\text { Bradycardia } \sqsubseteq \neg \text { HighBloodPressure, i〉\}, }
$$

over the lattice $L_{4}$ from Figure 1. The degree $\mathrm{i}$ in these axioms expresses that the relation between the causes and HighBloodPressure is not absolute. Consider the patients ana, who is a coffee drinker, and bob, a salt consumer with bradycardia, as expressed by the ABox

$$
\{\langle\text { ana : CoffeeDrinker }=\mathrm{t}\rangle,\langle\text { bob : SaltConsumer } \sqcap \text { Bradycardia }=\mathrm{t}\rangle\} .
$$

We can deduce that both patients are an i-instance of HighBloodPressure, but only bob is an i-instance of $\neg$ HighBloodPressure. Notice that if we changed all the degrees from the GCIs to the value $t$, the ontology would be inconsistent.

We will focus first on a version of the consistency problem where the ABox is required to be a local ABox; we call this problem local consistency. We show in Section 5 that local consistency can be used for solving other reasoning problems in $L-\mathcal{S H I}$ if $L$ is finite. Before that, we show that satisfiability and (local) consistency are undecidable in $L-\mathcal{A L C}$, and hence also in $L-\mathcal{S H I}$, in general. 


\section{Undecidability}

To show undecidability, we use a reduction from the Post Correspondence Problem [24] to strong satisfiability in $L-\mathcal{A L C}$ over a specific infinite lattice. The reduction uses ideas that have been successfully applied to showing undecidability of reasoning for several fuzzy description logics $[2,3,14]$.

Although the basic idea of the proof is not new, it is interesting for several reasons. First, previous incarnations of the proof idea focused on decidability of ontology consistency $[3,13,14]$, while we are concerned with strong $\ell$-satisfiability. Second, most of the previous undecidability results only hold for reasoning w.r.t. witnessed models, but the current proof works for both witnessed and general models. Finally, in contrast to an earlier version of this proof [11], the employed lattice has a quite simple structure in the sense that it is a total order that has only the two limit points $-\infty$ and $\infty$ instead of infinitely many. Note that any distributive lattice without limit points is already finite and reasoning in finite residuated De Morgan lattices is decidable (see Sections 4 and 5).

Definition 7 (PCP). Let $\mathcal{P}=\left\{\left(v_{1}, w_{1}\right), \ldots,\left(v_{n}, w_{n}\right)\right\}$ be a finite set of pairs of words over the alphabet $\Sigma=\{1, \ldots, s\}$ with $s>1$. The Post Correspondence Problem (PCP) asks for a finite non-empty sequence $i_{1} \ldots i_{k} \in\{1, \ldots, n\}^{+}$such that $v_{i_{1}} \ldots v_{i_{k}}=w_{i_{1}} \ldots w_{i_{k}}$. If this sequence exists, it is called a solution for $\mathcal{P}$.

For $\nu=i_{1} \cdots i_{k} \in\{1, \ldots, n\}^{*}$, we define $v_{\nu}:=v_{i_{1}} \cdots v_{i_{k}}$ and $w_{\nu}:=w_{i_{1}} \cdots w_{i_{k}}$.

We consider the lattice $\mathbb{Z}_{\infty}$ whose domain is $\mathbb{Z} \cup\{-\infty, \infty\}$ with the usual ordering over the integers and $-\infty$ and $\infty$ as the minimal and maximal element, respectively. Its De Morgan negation is $\sim \ell=-\ell$ if $\ell \in \mathbb{Z}, \sim \infty=-\infty$, and $\sim(-\infty)=\infty$. The t-norm $\otimes$ is defined as follows for all $\ell, m \in \mathbb{Z}_{\infty}$ :

$$
\ell \otimes m:= \begin{cases}\ell+m & \text { if } \ell, m \in \mathbb{Z} \text { and } \ell, m \leq 0 \\ \min \{\ell, m\} & \text { otherwise. }\end{cases}
$$

This is in fact a residuated lattice with the following residuum:

$$
\ell \Rightarrow m:= \begin{cases}\infty & \text { if } \ell \leq m \\ m & \text { if } \ell>m \text { and } \ell \geq 0 \\ m-\ell & \text { if } \ell>m \text { and } \ell<0 .\end{cases}
$$

Given an instance $\mathcal{P}$ of the PCP, we will construct a TBox $\mathcal{T}_{\mathcal{P}}$ such that the designated concept name $S$ is strongly $\infty$-satisfiable iff $\mathcal{P}$ has no solution. Recall that the alphabet $\Sigma$ consists of the first $s$ positive integers. Thus, every word in $\Sigma^{+}$can be seen as a positive integer written in base $s+1$; we extend this intuition and denote the empty word by 0 . We encode each word $u \in \Sigma^{*}$ with the number $-u \leq 0$.

The idea is that the TBox $\mathcal{T}_{\mathcal{P}}$ describes the search tree of $\mathcal{P}$ with the nodes $\{1, \ldots, n\}^{*}$. At its root $\varepsilon$, it encodes the value $v_{\varepsilon}=w_{\varepsilon}=\varepsilon$, which is represented by 0 , using the concept names $V$ and $W$. These concept names are used throughout the tree to express the values $v_{\nu}$ and $w_{\nu}$ at every node $\nu \in\{1, \ldots, n\}^{*}$. 
Additionally, we will use the auxiliary concept names $V_{i}$ and $W_{i}$ to encode the constant words $v_{i}$ and $w_{i}$, respectively, for each $i \in\{1, \ldots, n\}$. These will be used to compute the concatenation $v_{\nu i}=v_{\nu} v_{i}$ at each node.

To simplify the reduction, we will use some abbreviations. Given two $L-\mathcal{A L C}$ concepts $C$ and $D$ and $r \in \mathrm{N}_{\mathrm{R}},\langle C \equiv D\rangle$ abbreviates the axioms $\langle C \sqsubseteq D, \infty\rangle$, $\langle D \sqsubseteq C, \infty\rangle ;$ and $\langle C \stackrel{r}{\rightsquigarrow} D\rangle$ stands for the axioms $\langle C \sqsubseteq \forall r . D, \infty\rangle,\langle\exists r . D \sqsubseteq C, \infty\rangle$. For $n \geq 1$, the concept $C^{n}$ is inductively defined by $C^{1}:=C$ and $C^{n+1}:=C^{n} \sqcap C$.

Proposition 8. Let $\mathcal{I}$ be an interpretation and $x \in \Delta^{\mathcal{I}}$.

- If $\mathcal{I}$ satisfies $\langle C \equiv D\rangle$, then $C^{\mathcal{I}}(x)=D^{\mathcal{I}}(x)$.

- If I satisfies $\langle C \stackrel{r}{\rightsquigarrow} D\rangle$ and $C^{\mathcal{I}}(x) \leq 0$, then $C^{\mathcal{I}}(x)=D^{\mathcal{I}}(y)$ holds for all $y \in \Delta^{\mathcal{I}}$ with $r^{\mathcal{I}}(x, y) \geq 1$.

- If $C^{\mathcal{I}}(x) \in \mathbb{Z}, C^{\mathcal{I}}(x) \leq 0$, and $n \geq 1$, then $\left(C^{n}\right)^{\mathcal{I}}(x)=n \cdot C^{\mathcal{I}}(x)$.

We now introduce the TBox $\mathcal{T}_{0}:=\bigcup_{i=0}^{n} \mathcal{T}_{\mathcal{P}}^{i}$ that encodes the search tree of the instance $\mathcal{P}$ of the PCP:

$$
\begin{aligned}
\mathcal{T}_{\mathcal{P}}^{0}:= & \{\langle S \sqsubseteq V, 0\rangle,\langle S \sqsubseteq \neg V, 0\rangle,\langle S \sqsubseteq W, 0\rangle,\langle S \sqsubseteq \neg W, 0\rangle\}, \\
\mathcal{T}_{\mathcal{P}}^{i}:= & \left\{\left\langle\top \sqsubseteq \exists r_{i} \cdot \top, 1\right\rangle,\right. \\
& \left\langle\top \sqsubseteq V_{i},-v_{i}\right\rangle,\left\langle\top \sqsubseteq \neg V_{i}, v_{i}\right\rangle,\left\langle\top \sqsubseteq W_{i},-w_{i}\right\rangle,\left\langle\top \sqsubseteq \neg W_{i}, w_{i}\right\rangle, \\
& \left.\left\langle\left(V^{(s+1)^{\left|v_{i}\right|}} \sqcap V_{i}\right) \stackrel{r_{i}}{\sim} V\right\rangle,\left\langle\left(W^{(s+1)^{\left|w_{i}\right|}} \sqcap W_{i}\right) \stackrel{r_{i}}{\rightsquigarrow} W\right\rangle\right\},
\end{aligned}
$$

where $|u|$ denotes the length of the word $u$.

The TBox $\mathcal{T}_{\mathcal{P}}^{0}$ initializes the search tree by ensuring for every model $\mathcal{I}$ and every domain element $x \in \Delta^{\mathcal{I}}$ that satisfies $S^{\mathcal{I}}(x)=\infty$ that the values of $V$ and $W$ are both 0 , which is the encoding of the empty word. Each TBox $\mathcal{T}_{\mathcal{P}}^{i}$ ensures the existence of an $r_{i}$-successor for every domain element and describes the constant pair $\left(v_{i}, w_{i}\right)$ using the concepts $V_{i}$ and $W_{i}$, that is, it forces that $V_{i}^{\mathcal{I}}(x)=-v_{i}$ and $W_{i}^{\mathcal{I}}(x)=-w_{i}$ for every $x \in \Delta^{\mathcal{I}}$. Using the last two axioms, the search tree is then extended by concatenating the words $v$ and $w$ produced so far with $v_{i}$ and $w_{i}$, respectively. In the following, we will describe this in more detail.

Consider the interpretation $\mathcal{I}_{\mathcal{P}}$ over the domain $\Delta^{\mathcal{I}_{\mathcal{P}}}=\{1, \ldots, n\}^{*}$, where for all $\nu, \nu^{\prime} \in\{1, \ldots, n\}^{*}$ and $i \in\{1, \ldots, n\}$,

$$
\begin{aligned}
& -V^{\mathcal{I}_{\mathcal{P}}}(\nu)=-v_{\nu}, \quad W^{\mathcal{I}_{\mathcal{P}}}(\nu)=-w_{\nu}, \\
& -V_{i}^{\mathcal{I}_{\mathcal{P}}}(\nu)=-v_{i}, \quad W_{i}^{\mathcal{I}_{\mathcal{P}}}(\nu)=-w_{i}, \\
& -r_{i}^{\mathcal{I}_{\mathcal{P}}}(\nu, \nu i)=\infty \text { and } r_{i}^{\mathcal{I}_{\mathcal{P}}}\left(\nu, \nu^{\prime}\right)=-\infty \text { if } \nu^{\prime} \neq \nu i, \\
& -S^{\mathcal{I}_{\mathcal{P}}}(\varepsilon)=\infty \text { and } S^{\mathcal{I}_{\mathcal{P}}}\left(\nu^{\prime}\right)=-\infty \text { if } \nu^{\prime} \neq \varepsilon .
\end{aligned}
$$

It is easy to see that $\mathcal{I}_{\mathcal{P}}$ is in fact a model of $\mathcal{T}_{0}$ and it strongly satisfies $S$ with degree $\infty$. Moreover, every model of this TBox that strongly $\infty$-satisfies $S$ must "include" $\mathcal{I}_{\mathcal{P}}$ in the following sense.

Lemma 9. Let $\mathcal{I}$ be a model of $\mathcal{T}_{0}$ such that $S^{\mathcal{I}}\left(x_{0}\right)=\infty$ for some $x_{0} \in \Delta^{\mathcal{I}}$. Then there exists a function $g: \Delta^{\mathcal{I}_{\mathcal{P}}} \rightarrow \Delta^{\mathcal{I}}$ such that $A^{\mathcal{I}_{\mathcal{P}}}(\nu)=A^{\mathcal{I}}(g(\nu))$ and $r_{i}(g(\nu), g(\nu i)) \geq 1$ hold for every concept name $A \in\left\{V, W, V_{1}, W_{1}, \ldots, V_{n}, W_{n}\right\}$, every $\nu \in \Delta^{\mathcal{I}_{\mathcal{P}}}$, and every $i \in\{1, \ldots, n\}$. 
Proof. We construct the function $g$ by induction on $\nu$ and set $g(\varepsilon):=x_{0}$. Since $\mathcal{I}$ is a model of $\mathcal{T}_{\mathcal{P}}^{0}$ and $S^{\mathcal{I}}\left(x_{0}\right)=\infty$, we have $V^{\mathcal{I}}\left(x_{0}\right) \geq 0$ and $\sim V^{\mathcal{I}}\left(x_{0}\right) \geq 0$, i.e. $V^{\mathcal{I}}\left(x_{0}\right)=0$, and similarly $W^{\mathcal{I}}\left(x_{0}\right)=0$. In the same way, for every $i \in\{1, \ldots, n\}$, $V_{i}^{\mathcal{I}}\left(x_{0}\right)$ and $W_{i}^{\mathcal{I}}\left(x_{0}\right)$ are restricted by $\mathcal{T}_{\mathcal{P}}^{i}$ to be $-v_{i}$ and $-w_{i}$, respectively.

Let now $\nu \in\{1, \ldots, n\}^{*}$ and assume that $g(\nu)$ already satisfies the condition. For each $i \in\{1, \ldots, n\}$, the first axiom of $\mathcal{T}_{\mathcal{P}}^{i}$ ensures that $\bigvee_{y \in \Delta^{\mathcal{I}}} r_{i}^{\mathcal{I}}(g(\nu), y) \geq 1$. Thus, there is $y_{i} \in \Delta^{\mathcal{I}}$ such that $r_{i}^{\mathcal{I}}\left(g(\nu), y_{i}\right) \geq 1$. We define $g(\nu i):=y_{i}$. By Proposition 8, we have

$$
V^{\mathcal{I}}\left(y_{i}\right)=\left(V^{(s+1)^{\left|v_{i}\right|}} \sqcap V_{i}\right)^{\mathcal{I}}(g(\nu))=-\left((s+1)^{\left|v_{i}\right|} v_{\nu}+v_{i}\right)=-v_{\nu} v_{i}=-v_{\nu i},
$$

and similarly for $W^{\mathcal{I}}\left(y_{i}\right)$. The claim for $V_{i}$ and $W_{i}$ can be shown as above.

This proposition shows that every model of $\mathcal{T}_{0}$ encodes a description of the search tree for a solution of $\mathcal{P}$. Thus, to decide the PCP, it suffices to detect whether there is a node $\nu \in\{1, \ldots, n\}^{+}$of $\mathcal{I}_{\mathcal{P}}$ where $V^{\mathcal{I}_{\mathcal{P}}}(\nu)=W^{\mathcal{I}_{\mathcal{P}}}(\nu)$. We accomplish this using the TBox

$$
\mathcal{T}^{\prime}:=\left\{\left\langle\top \sqsubseteq \forall r_{i} . \neg((V \rightarrow W) \sqcap(W \rightarrow V)), 0\right\rangle \mid 1 \leq i \leq n\right\} .
$$

The interpretation $\mathcal{I}_{\mathcal{P}}$ is a model of $\mathcal{T}^{\prime}$ iff $V^{\mathcal{I}_{\mathcal{P}}}(\nu) \neq W^{\mathcal{I}_{\mathcal{P}}}(\nu)$ holds for every $\nu \in\{1, \ldots, n\}^{+}$.

Lemma 10. $\mathcal{P}$ has a solution iff $S$ is not $\infty$-satisfiable w.r.t. $\mathcal{T}_{\mathcal{P}}:=\mathcal{T}_{0} \cup \mathcal{T}^{\prime}$.

Proof. For any two values $\ell, m \leq 0$, we have $\ell \neq m$ iff $(\ell \Rightarrow m) \otimes(m \Rightarrow \ell) \leq 0$.

Assume now that $S$ is not $\infty$-satisfiable w.r.t. $\mathcal{T}_{\mathcal{P}}$. Then, in particular, $\mathcal{I}_{\mathcal{P}}$ does not satisfy $\mathcal{T}^{\prime}$, i.e. we have $\left(\forall r_{i} . \neg((V \rightarrow W) \sqcap(W \rightarrow V))\right)^{\mathcal{I}_{\mathcal{P}}}(\nu)<0$ for some $\nu \in\{1, \ldots, n\}^{*}$ and $i \in\{1, \ldots, n\}$. There must be a $\nu \in\{1, \ldots, n\}^{+}$with $(\neg((V \rightarrow W) \sqcap(W \rightarrow V)))^{\mathcal{I}_{\mathcal{P}}}(\nu)<0$; thus, $-v_{\nu}=V^{\mathcal{I}_{\mathcal{P}}}(\nu)=W^{\mathcal{I}_{\mathcal{P}}}(\nu)=-w_{\nu}$. This shows that $v_{\nu}=w_{\nu}$, i.e. $\mathcal{P}$ has a solution.

For the other direction, let $\mathcal{I}$ be a model of $\mathcal{T}_{\mathcal{P}}$ and $x_{0} \in \Delta^{\mathcal{I}}$ such that $S^{\mathcal{I}}\left(x_{0}\right)=\infty$. In particular, we have

$$
r_{i}^{\mathcal{I}}(g(\nu), g(\nu i)) \Rightarrow(\neg((V \rightarrow W) \sqcap(W \rightarrow V)))^{\mathcal{I}}(g(\nu i)) \geq 0
$$

for every $\nu \in\{1, \ldots, n\}^{*}$ and $i \in\{1, \ldots, n\}$, where $g$ is the function constructed in Lemma 9. Thus, $((V \rightarrow W) \sqcap(W \rightarrow V))^{\mathcal{I}}(g(\nu)) \leq 0$ for every $\nu \in\{1, \ldots, n\}^{+}$, which implies $-v_{\nu}=V^{\mathcal{I}}(g(\nu)) \neq W^{\mathcal{I}}(g(\nu))=-w_{\nu}$. This shows that $v_{\nu} \neq w_{\nu}$ for all $\nu \in\{1, \ldots, n\}^{+}$, i.e. $\mathcal{P}$ has no solution.

As mentioned before, since the interpretation $\mathcal{I}_{\mathcal{P}}$ is witnessed, undecidability holds even if we restrict reasoning to $n$-witnessed models, for any $n \in \mathbb{N}$.

Theorem 11. Strong satisfiability is undecidable in $L-\mathcal{A L C}$, for some countable total order $L$ with at most two limit points, even if reasoning is over $n$-witnessed models only. 
This theorem also shows that (local) consistency is undecidable in $\mathbb{Z}_{\infty}-\mathcal{A L C}$ since $S$ is strongly $\infty$-satisfiable w.r.t. $\mathcal{T}_{\mathcal{P}}$ iff $\left(\{\langle a: S=\infty\rangle\}, \mathcal{T}_{\mathcal{P}}\right)$ is locally consistent, where $a$ is an arbitrary individual name.

Notice that this does not exclude the existence of classes of infinite lattices for which reasoning in $L-\mathcal{S H I}$ is decidable. In fact, there exists a large class of infinite total orders for which consistency is decidable [9]. What Theorem 11 shows is that there exist lattices for which this problem is undecidable. If we restrict to finite lattices, then a tableau algorithm can be used for reasoning.

\section{A Tableaux Algorithm for Local Consistency}

Before presenting a tableau algorithm [4] that decides local consistency by constructing a model of a given $L-\mathcal{S H I}$ ontology, we discuss previous approaches to deciding consistency of fuzzy DLs over finite residuated De Morgan lattices in the presence of GCIs.

A popular method is the reduction of fuzzy ontologies into crisp ones, which has so far only been done for finite total orders [7,8,26]. Reasoning can then be performed through existing optimized reasoners for crisp DLs. The main idea is to translate every concept name $A$ into finitely many crisp concept names $A_{\geq \ell}$, one for each truth value $\ell$, where $A_{\geq \ell}$ collects all those individuals that belong to $A$ with a truth degree $\geq \ell$. The lattice structure is expressed through GCIs of the form $A_{\geq \ell_{2}} \sqsubseteq A_{\geq \ell_{1}}$, where $\ell_{2}$ is a minimal element above $\ell_{1}$, and analogously for the role names. All axioms are then recursively translated into crisp axioms that use only the introduced crisp concept and role names. The resulting crisp ontology is consistent iff the original fuzzy ontology is consistent.

In general such a translation is exponential in the size of the concepts that occur in the fuzzy ontology. The reason is that, depending on the t-norm used, there may be many possible combinations of values $\ell_{1}, \ell_{2}$ for $C, D$, respectively, that lead to $C \sqcap D$ having the value $\ell=\ell_{1} \otimes \ell_{2}$, and similarly for the other constructors. All these possibilities have to be expressed in the translation. Since ontology consistency in crisp $\mathcal{S H \mathcal { I }}$ is ExPTIME-hard, this yields a 2-ExPTIME reasoning procedure. Moreover, DL reasoners usually implement tableaux algorithms with a worst-case complexity above NEXPTimE; in that case, one gets a 2-NEXPTIME reasoning procedure. In contrast, our tableau algorithm has a worst-case complexity of NExPTime, matching the behaviour of crisp $\mathcal{S H \mathcal { I }}$ reasoners.

To the best of our knowledge, at the moment there exists only one (correct) tableaux algorithm that can deal with a finite total order of truth values and GCIs $[25],{ }^{3}$ but it is restricted to the Gödel t-norm. The main difference between this algorithm and ours is that we non-deterministically guess the degree of membership of each individual to every relevant concept, while the approach from [25] sets only lower and upper bounds for these degrees; this greatly reduces

\footnotetext{
${ }^{3}$ Several tableau algorithms for fuzzy DLs over infinite total orders exist, but they are either restricted to acyclic TBoxes or are not correct in the presence of GCIs, as shown in $[2,6]$.
} 
the amount of non-determinism encountered, but introduces several complications when a t-norm different from the Gödel t-norm is used.

We present a straightforward tableaux algorithm with a larger amount of nondeterminism that nevertheless matches the theoretical worst-case complexity of tableaux algorithms for crisp $\mathcal{S H \mathcal { I }}$. It is loosely based on the crisp tableaux algorithm in [20]. A first observation that simplifies the algorithm is that since $L$ is finite, we can w.l.o.g. restrict reasoning to $n$-witnessed models.

Proposition 12. If the maximal cardinality of an antichain of $L$ is $n$, then every interpretation in $L-\mathcal{S H I}$ is $n$-witnessed.

For simplicity, we consider only the case $n=1$. For $n>1$, the construction is similar, but several witnesses have to be produced for satisfying each existential and value restriction. The necessary changes in the algorithm are described at the end of this section. We can also assume w.l.o.g. that the RBox is acyclic. The proof of this follows similar arguments as for crisp $\mathcal{S H \mathcal { I }}[27]$.

Proposition 13. Deciding local consistency in $L-\mathcal{S H I}$ is polynomially equivalent to deciding local consistency in $L-\mathcal{S H I}$ w.r.t. acyclic RBoxes.

In the following, let $\mathcal{O}=(\mathcal{A}, \mathcal{T}, \mathcal{R})$ be an ontology where $\mathcal{A}$ is a local $\mathrm{ABox}$ that contains only the individual name $a$ and $\mathcal{R}$ is an acyclic RBox. We first show that $\mathcal{O}$ has a model if we can find a tableau; intuitively, a possibly infinite "completed version" of $\mathcal{A}$. Later we describe an algorithm for constructing a finite representation of such a tableau.

Definition 14. A tableau for $\mathcal{O}$ is a set $\mathbf{T}$ of equality assertions over a set Ind of individuals such that $a \in \operatorname{Ind}, \mathcal{A} \subseteq \mathbf{T}$, and the following conditions are satisfied for all $C, C_{1}, C_{2} \in \operatorname{sub}(\mathcal{O}), x, y \in$ Ind, $r, s \in \mathrm{N}_{\mathrm{R}}$, and $\ell \in L$ :

Clash-free: If $\langle x: C=\ell\rangle \in \mathbf{T}$ or $\langle(x, y): r=\ell\rangle \in \mathbf{T}$, then there is no $\ell^{\prime} \in L$ such that $\ell^{\prime} \neq \ell$ and $\left\langle x: C=\ell^{\prime}\right\rangle \in \mathbf{T}$ or $\left\langle(x, y): r=\ell^{\prime}\right\rangle \in \mathbf{T}$, respectively.

Complete: For every row of Table 1, the following condition holds:

"If 〈trigger〉 is in $\mathbf{T}$, there are 〈values〉 such that 〈assertions〉 are in $\mathbf{T}$."

These conditions help to abstract from the interplay between transitive roles and existential and value restrictions. We prove in [12] that it suffices to satisfy the above conditions to make certain that $\mathcal{O}$ has a model.

Lemma 15. $\mathcal{O}$ is locally consistent iff it has a tableau.

We now present a tableaux algorithm for deciding local consistency. The algorithm starts with the local ABox $\mathcal{A}$, and nondeterministically expands it to a tree-like ABox $\widehat{\mathcal{A}}$ that represents a model of $\mathcal{O}$. It uses the conditions from Table 1 and reformulates them into expansion rules of the form:

"If there is 〈trigger〉 in $\widehat{\mathcal{A}}$ and there are no 〈values〉 such that 〈assertions〉 are in $\mathcal{A}$, then introduce $\langle$ values $\rangle$ and add 〈assertions $\rangle$ to $\widehat{\mathcal{A}}$." 


\begin{tabular}{|c|c|c|c|}
\hline & $\mid\langle$ trigger $\rangle$ & $\langle$ values $\rangle$ & $\langle$ assertions $\rangle$ \\
\hline$\Pi$ & $\left\langle\left\langle x: C_{1} \sqcap C_{2}=\ell\right\rangle\right.$ & $\ell_{1}, \ell_{2} \in L$ with $\ell_{1} \otimes \ell_{2}=\ell$ & $\begin{array}{l}\left\langle x: C_{1}=\ell_{1}\right\rangle \\
\left\langle x: C_{2}=\ell_{2}\right\rangle\end{array}$ \\
\hline$\sqcup$ & $\left\langle x: C_{1} \sqcup C_{2}=\ell\right\rangle$ & $\ell_{1}, \ell_{2} \in L$ with $\ell_{1} \oplus \ell_{2}=\ell$ & $\begin{array}{l}\left\langle x: C_{1}=\ell_{1}\right\rangle \\
\left\langle x: C_{2}=\ell_{2}\right\rangle\end{array}$ \\
\hline$\rightarrow$ & $\left\langle x: C_{1} \rightarrow C_{2}=\ell\right\rangle$ & $\ell_{1}, \ell_{2} \in L$ with $\ell_{1} \Rightarrow \ell_{2}=\ell$ & $\begin{array}{l}\left\langle x: C_{1}=\ell_{1}\right\rangle \\
\left\langle x: C_{2}=\ell_{2}\right\rangle\end{array}$ \\
\hline$\neg$ & $\langle x: \neg C=\ell\rangle$ & & $\langle x: C=\sim \ell\rangle$ \\
\hline$\exists$ & $\langle x: \exists r . C=\ell\rangle$ & $\begin{array}{l}\ell_{1}, \ell_{2} \in L \text { with } \ell_{1} \otimes \ell_{2}=\ell, \\
\text { individual } y\end{array}$ & $\begin{array}{l}\left\langle(x, y): r=\ell_{1}\right\rangle \\
\left\langle y: C=\ell_{2}\right\rangle\end{array}$ \\
\hline$\exists_{\leq}$ & $\langle x: \exists r . C=\ell\rangle,\left\langle(x, y): r=\ell_{1}\right\rangle$ & $\ell_{2} \in L$ with $\ell_{1} \otimes \ell_{2} \leq \ell$ & $\left\langle y: C=\ell_{2}\right\rangle$ \\
\hline$\exists_{+}$ & $\begin{array}{l}\langle x: \exists s . C=\ell\rangle,\left\langle(x, y): r=\ell_{1}\right\rangle \\
\text { with } r \text { transitive and } r \sqsubseteq \mathcal{R} s\end{array}$ & $\ell_{2} \in L$ with $\ell_{1} \otimes \ell_{2} \leq \ell$ & $\left\langle y: \exists r . C=\ell_{2}\right\rangle$ \\
\hline$\forall$ & $\langle x: \forall r . C=\ell\rangle$ & $\begin{array}{l}\ell_{1}, \ell_{2} \in L \text { with } \ell_{1} \Rightarrow \ell_{2}=\ell \\
\text { individual } y\end{array}$ & $\begin{array}{l}\left\langle(x, y): r=\ell_{1}\right\rangle \\
\left\langle y: C=\ell_{2}\right\rangle\end{array}$ \\
\hline$\forall \geq$ & $\langle x: \forall r . C=\ell\rangle,\left\langle(x, y): r=\ell_{1}\right\rangle$ & $\ell_{2} \in L$ with $\ell_{1} \Rightarrow \ell_{2} \geq \ell$ & $\left\langle y: C=\ell_{2}\right\rangle$ \\
\hline$\forall_{+}$ & $\begin{array}{l}\langle x: \forall s . C=\ell\rangle,\left\langle(x, y): r=\ell_{1}\right\rangle \\
\text { with } r \text { transitive and } r \sqsubseteq \mathcal{R} s\end{array}$ & $\ell_{2} \in L$ with $\ell_{1} \Rightarrow \ell_{2} \geq \ell$ & $\left\langle y: \forall r \cdot C=\ell_{2}\right\rangle$ \\
\hline inv & $\left\langle(x, y): r=\ell_{1}\right\rangle$ & & $\left\langle(y, x): \bar{r}=\ell_{1}\right\rangle$ \\
\hline$\sqsubseteq \mathcal{R}$ & $\left\langle(x, y): r=\ell_{1}\right\rangle, r \sqsubseteq \mathcal{R} s$ & $\ell_{2} \in L$ with $\ell_{1} \leq \ell_{2}$ & $\left\langle(x, y): s=\ell_{2}\right\rangle$ \\
\hline$\sqsubseteq \mathcal{T}$ & individual $x,\left\langle C_{1} \sqsubseteq C_{2}, \ell\right\rangle$ in $\mathcal{T}$ & $\ell_{1}, \ell_{2} \in L$ with $\ell_{1} \Rightarrow \ell_{2} \geq \ell$ & $\begin{array}{l}\left\langle x: C_{1}=\ell_{1}\right\rangle \\
\left\langle x: C_{2}=\ell_{2}\right\rangle\end{array}$ \\
\hline
\end{tabular}

Table 1. The tableau conditions for $L-\mathcal{S H I}$.

The rules $\exists$ and $\forall$ always introduce new individuals $y$ that do not appear in $\widehat{\mathcal{A}}$. Initially, the $\operatorname{ABox} \mathcal{A}$ contains the single individual $a$. It is expanded by the rules in a tree-like way: role connections are only created by adding new successors to existing individuals. If an individual $y$ was created by a rule $\exists$ or $\forall$ that was applied to an assertion involving an individual $x$, then we say that $y$ is a successor of $x$, and $x$ is the predecessor of $y$; ancestor is the transitive closure of predecessor. Note that the presence of an assertion $\langle(x, y): r=\ell\rangle$ in $\widehat{\mathcal{A}}$ does not imply that $y$ is a successor of $x$-it could also be the case that this assertion was introduced by the inv-rule. We further denote by $\widehat{\mathcal{A}}_{x}$ the set of all concept assertions from $\widehat{\mathcal{A}}$ that involve the individual $x$, i.e. are of the form $\langle x: C=\ell\rangle$ for some concept $C$ and $\ell \in L$. To ensure that the application of the rules terminates, we need to add a blocking condition. We use anywhere blocking [23], which is based on the idea that it suffices to examine each set $\widehat{\mathcal{A}}_{x}$ only once in the whole ABox $\widehat{\mathcal{A}}$.

Let $\succ$ be a total order on the individuals of $\widehat{\mathcal{A}}$ that includes the ancestor relationship, i.e. whenever $y$ is a successor of $x$, then $y \succ x$. An individual $y$ is directly blocked if for some other individual $x$ in $\widehat{\mathcal{A}}$ with $y \succ x, \widehat{\mathcal{A}}_{x}$ is equal to $\widehat{\mathcal{A}}_{y}$ modulo the individual names; in this case, we write $\widehat{\mathcal{A}}_{x} \equiv \widehat{\mathcal{A}}_{y}$ and also say that $x$ blocks $y$. It is indirectly blocked if its predecessor is either directly or indirectly blocked. A node is blocked if it is either directly or indirectly blocked. The rules 
$\exists$ and $\forall$ are applied to $\widehat{\mathcal{A}}$ only if the node $x$ that triggers their execution is not blocked. All other rules are applied only if $x$ is not indirectly blocked.

The total order $\succ$ avoids cycles in the blocking relation. One possibility is to simply use the order in which the individuals were created by the expansion rules. Note that the only individual $a$ that occurs in $\mathcal{A}$, which is the root of the tree-like structure represented by $\widehat{\mathcal{A}}$, cannot be blocked since it is an ancestor of all other individuals in $\widehat{\mathcal{A}}$. With this blocking condition, we can show that the size of $\widehat{\mathcal{A}}$ is bounded exponentially in the size of $\mathcal{A}$, as in the crisp case [23].

Lemma 16. Every application of expansion rules to $\mathcal{A}$ terminates after at most exponentially many rule applications.

We say that $\widehat{\mathcal{A}}$ contains a clash if it contains two assertions that are equal except for their lattice value (see Definition 14). $\widehat{\mathcal{A}}$ is complete if it contains a clash or none of the expansion rules are applicable. The algorithm is correct in the sense that it produces a clash iff $\mathcal{O}$ is not locally consistent (see [12] for details).

Lemma 17. $\mathcal{O}$ is locally consistent iff some application of the expansion rules to $\mathcal{A}$ yields a complete and clash-free ABox.

Since the tableau rules are nondeterministic, Lemmata 16 and 17 together imply that the tableaux algorithm decides local consistency in NEXPTIME.

Theorem 18. Local consistency in L-SHI w.r.t. witnessed models can be decided in NEXPTIME.

As explained before, $L-\mathcal{S H I}$ has the $n$-witnessed model property for some $n \geq 1$. We have so far restricted our description to the case where $n=1$. If $n>1$, it does not suffice to generate only one successor for every existential and universal restriction, but one must produce $n$ different successors to ensure that the degrees guessed for these complex concepts are indeed witnessed by the model. The only required change to the algorithm is in the rows $\exists$ and $\forall$ of Table 1 , where we have to introduce $n$ individuals $y_{1}, \ldots, y_{n}$, and $2 n$ values $\ell_{1}^{1}, \ell_{2}^{1}, \ldots, \ell_{1}^{n}, \ell_{2}^{n} \in L$ that satisfy $\bigvee_{i=1}^{n} \ell_{1}^{i} \otimes \ell_{2}^{i}=\ell$ or $\bigwedge_{i=1}^{n} \ell_{1}^{i} \Rightarrow \ell_{2}^{i}=\ell$, respectively.

\section{Local Completion and Other Black-Box Reductions}

In the following, we assume that we have a black-box procedure that decides local consistency in a sublogic of $L-\mathcal{S H \mathcal { I }}$. This procedure can be, e.g. the tableaubased algorithm from the previous section, or any other method for solving this decision problem. We show how to employ such a procedure to solve other reasoning problems for this sublogic.

\subsection{Consistency}

To reduce consistency of an arbitrary ontology $\mathcal{O}=(\mathcal{A}, \mathcal{T}, \mathcal{R})$ to local consistency, we first make sure that the information contained in $\mathcal{A}$ is consistent "in 
itself", i.e. if we only consider the individuals occurring in $\mathcal{A}$. It then suffices to check a local consistency condition for each of the individuals.

Let $\operatorname{Ind}_{\mathcal{A}}$ denote the set of individual names occurring in $\mathcal{A}$ and $\operatorname{sub}(\mathcal{A}, \mathcal{T})$ the set of all subconcepts of concepts occurring in $\mathcal{A}$ or $\mathcal{T}$. We first guess a set $\widehat{\mathcal{A}}$ of equality assertions of the forms $\langle a: C=\ell\rangle$ and $\langle(a, b): r=\ell\rangle$ with $a, b \in \operatorname{Ind}_{\mathcal{A}}$, $C \in \operatorname{sub}(\mathcal{A}, \mathcal{T}), r \in \mathrm{N}_{\mathrm{R}}$, and $\ell \in L$. We then check whether $\widehat{\mathcal{A}}$ is clash-free and satisfies the tableau conditions listed in Table 1, except the witnessing conditions $\exists$ and $\forall$. Additionally, we impose the following condition on $\widehat{\mathcal{A}}$ :

"If there is an assertion $\langle\alpha \triangleright \ell\rangle$ in $\mathcal{A}$, then there is $\ell^{\prime} \in L$ such that $\ell^{\prime} \triangleright \ell$ and $\left\langle\alpha=\ell^{\prime}\right\rangle$ is in $\widehat{\mathcal{A}}$."

We call $\widehat{\mathcal{A}}$ locally complete iff it is of the above form and satisfies all of the above conditions. Guessing this set and checking whether it is locally complete can be done in polynomial time in the size of $\mathcal{O}$.

Lemma 19. An ontology $\mathcal{O}=(\mathcal{A}, \mathcal{T}, \mathcal{R})$ is consistent iff there is a locally complete set $\widehat{\mathcal{A}}$ such that $\mathcal{O}_{x}=\left(\widehat{\mathcal{A}}_{x}, \mathcal{T}, \mathcal{R}\right)$ is locally consistent for every $x \in \operatorname{Ind}_{\mathcal{A}}$.

The proof of this lemma can be found in [12] and uses similar methods as the proofs for the results of the previous section.

Theorem 20. If local consistency in $L-\mathcal{S H I}$ can be decided in a complexity class $\mathrm{C}$, then consistency in $L-\mathcal{S H I}$ can be decided in any complexity class that contains both NP and $\mathrm{C}$.

This means that consistency in $L-\mathcal{S H \mathcal { I }}$ is decidable in NExPTime. In [10], an automata-based algorithm was presented that can decide satisfiability and subsumption in $L-\mathcal{A L C I}$ in ExpTime. Moreover, if the TBox is acyclic, then this bound can be improved to PSPACE. The algorithm can easily be adapted to decide local consistency. With the above reduction, this shows that consistency in $L-\mathcal{A L C I}$ w.r.t. general and acyclic TBoxes can be decided in ExpTime and PSPACE, respectively. The same argument applies to any sublogic of $L-\mathcal{S H I}$ for which local consistency can be decided in EXPTime or PSPACE.

\subsection{Satisfiability, Instance Checking, and Subsumption}

To decide whether a concept $C$ is strongly $\ell$-satisfiable w.r.t. $\mathcal{O}=(\mathcal{A}, \mathcal{T}, \mathcal{R})$, we can simply check whether $(\mathcal{A} \cup\{a: C \geq \ell\}, \mathcal{T}, \mathcal{R})$ is consistent for a new individual name $a$ not occurring in $\mathcal{A}$. Thus, strong $\ell$-satisfiability is in the same complexity class as consistency. Moreover, we can easily compute the set of all values $\ell \in L$ such that the ontology $(\mathcal{A} \cup\{a: C \geq \ell\}, \mathcal{T}, \mathcal{R})$ is consistent by calling the decision procedure for consistency a constant number of times, i.e. once for each $\ell \in L$. We can use this set to compute the best bound for the satisfiability of $C$. Formally, the best satisfiability degree of a concept $C$ is the supremum of all $\ell \in L$ such that $C$ is $\ell$-satisfiable w.r.t. $\mathcal{O}$. Since we can compute the set of all elements of $L$ satisfying this property, obtaining the best satisfiability degree 
requires only a supremum computation. As the lattice $L$ is fixed, this adds a constant factor to the complexity of checking consistency.

To check $\ell$-instances, we can exploit the fact that $a$ is not an $\ell$-instance of $C$ w.r.t. $\mathcal{O}$ iff there is a model $\mathcal{I}$ of $\mathcal{O}$ and a domain element $x \in \Delta^{\mathcal{I}}$ such that $C^{\mathcal{I}}\left(a^{\mathcal{I}}\right) \nsupseteq \ell$. This is the case iff there is a value $\ell^{\prime} \nsucceq \ell$ such that the ontology $\left(\mathcal{A} \cup\left\{a: C=\ell^{\prime}\right\}, \mathcal{T}, \mathcal{R}\right)$ is consistent. Thus, $\ell$-instances can be decided by calling the decision procedure for consistency a constant number of times, namely at most once for each $\ell^{\prime} \in L$ with $\ell^{\prime} \nsucceq \ell$. We can also compute the best instance degree for $a$ and $C$, which is the supremum of all $\ell \in L$ such that $a$ is an $\ell$-instance of $C$ w.r.t. $\mathcal{O}$. Let $\mathfrak{L}$ denote the set of all $\ell^{\prime}$ such that $\left(\left\{a: C=\ell^{\prime}\right\}, \mathcal{T}, \mathcal{R}\right)$ is consistent. The best instance degree for $a$ and $C$ is the infimum of all $\ell^{\prime} \in \mathfrak{L}$ since

$$
\begin{aligned}
\bigvee\{\ell \in L \mid a \text { is an } \ell \text {-instance of } C\} & =\bigvee\left\{\ell \in L \mid \forall \ell^{\prime} ¥ \ell: \ell^{\prime} \notin \mathfrak{L}\right\} \\
& =\bigvee\left\{\ell \in L \mid \forall \ell^{\prime} \in \mathfrak{L}: \ell \leq \ell^{\prime}\right\}=\bigwedge \mathfrak{L} .
\end{aligned}
$$

Finally, note that $C$ is $\ell$-subsumed by $D$ iff $a$ is an $\ell$-instance of $C \rightarrow D$, where $a$ is a new individual name. Thus, deciding $\ell$-subsumption and computing the best subsumption degree can be done using the same approach as above.

Lemma 21. If local consistency in $L-\mathcal{S H I}$ can be decided in a complexity class $\mathrm{C}$, then strong satisfiability, instance checking, and subsumption in $L-\mathcal{S H I}$ can be decided in any complexity class that contains both NP and C.

This shows that strong satisfiability, instance checking, and subsumption in $L-\mathcal{S H I}$ are in NExpTime. This bound reduces to ExpTime or PSPACE if we consider $L-\mathcal{A L C I}$ w.r.t. general or acyclic TBoxes, respectively [10].

\section{Conclusions}

We have studied fuzzy description logics with semantics based on complete residuated De Morgan lattices. We showed that even for the fairly inexpressive DL $L-\mathcal{A L C}$, strong satisfiability w.r.t. general TBoxes is undecidable for some infinite lattices. For finite lattices, decidability is regained. In fact, local consistency can be decided with a nondeterministic tableaux-based procedure in exponential time. We conjecture that this upper bound can be improved to ExPTIME either by an automata-based algorithm or with the help of advanced caching techniques [16]. However, automata-based approaches [10] can only deal with local consistency and concept satisfiability.

Our reduction shows that any algorithm deciding local consistency suffices for deciding consistency of ontologies, through the tableau-based local completion described in Section 5.1. In particular, this yields tight complexity bounds for deciding consistency in $L-\mathcal{A L C I}$ w.r.t. acyclic and general TBoxes-PSPACE and ExpTime, respectively. Other decision and computation problems can also be solved using a local consistency reasoner as a black box. 
The presented tableaux algorithm has highly nondeterministic rules, and as such is unsuitable for an implementation. Most of the optimizations developed for tableaux algorithms for crisp DLs, like the use of an optimized rule-application ordering, can be transfered to our setting. However, the most important task is to reduce the search space created by the choice of lattice values in most of the rules. We plan to study these optimizations in the future.

\section{References}

1. Baader, F., Calvanese, D., McGuinness, D.L., Nardi, D., Patel-Schneider, P.F. (eds.): The Description Logic Handbook: Theory, Implementation, and Applications. Cambridge University Press, 2nd edn. (2007)

2. Baader, F., Peñaloza, R.: Are fuzzy description logics with general concept inclusion axioms decidable? In: Proc. FUZZ-IEEE'11. pp. 1735-1742 (2011)

3. Baader, F., Peñaloza, R.: On the undecidability of fuzzy description logics with GCIs and product t-norm. In: Proc. FroCoS'11, LNCS, vol. 6989, pp. 55-70 (2011)

4. Baader, F., Sattler, U.: An overview of tableau algorithms for description logics. Studia Logica 69(1), 5-40 (2001)

5. Belnap, N.D.: A useful four-valued logic. In: Modern Uses of Multiple-Valued Logic, pp. 7-37. Reidel Publishing Company (1977)

6. Bobillo, F., Bou, F., Straccia, U.: On the failure of the finite model property in some fuzzy description logics. Fuzzy Set. Syst. 172(1), 1-12 (2011)

7. Bobillo, F., Straccia, U.: Finite fuzzy description logics: A crisp representation for finite fuzzy $\mathcal{A} \mathcal{L C H}$. In: Proc. URSW'10. CEUR, vol. 654, pp. 61-72 (2010)

8. Bobillo, F., Straccia, U.: Reasoning with the finitely many-valued Łukasiewicz fuzzy description logic $\mathcal{S} \mathcal{R O I} \mathcal{I}$. Inform. Sciences 181, 758-778 (2011)

9. Borgwardt, S., Distel, F., Peñaloza, R.: How fuzzy is my fuzzy description logic? In: Proc. IJCAR'12. LNAI, vol. 7364, pp. 82-96. Springer-Verlag (2012)

10. Borgwardt, S., Peñaloza, R.: Finite lattices do not make reasoning in $\mathcal{A} \mathcal{L C} \mathcal{I}$ harder. In: URSW'11. CEUR, vol. 778, pp. 51-62 (2011)

11. Borgwardt, S., Peñaloza, R.: Fuzzy ontologies over lattices with t-norms. In: Proc. DL'11. vol. 745, pp. 70-80. CEUR (2011)

12. Borgwardt, S., Peñaloza, R.: Consistency in fuzzy description logics over residuated De Morgan lattices. LTCS-Report 12-04, Chair for Automata Theory, TU Dresden, Germany (2012), see http://lat.inf.tu-dresden.de/research/reports.html.

13. Borgwardt, S., Peñaloza, R.: Undecidability of fuzzy description logics. In: Proc. KR'12. AAAI Press (2012)

14. Cerami, M., Straccia, U.: On the undecidability of fuzzy description logics with GCIs with Łukasiewicz $t$-norm. Tech. rep. (2011), arXiv:1107.4212v3 [cs.LO]

15. De Cooman, G., Kerre, E.E.: Order norms on bounded partially ordered sets. J. of Fuzzy Math. 2, 281-310 (1993)

16. Goré, R., Nguyen, L.A.: EXPTIME tableaux with global caching for description logics with transitive roles, inverse roles and role hierarchies. In: Proc. TABLEAUX'07. LNCS, vol. 4548, pp. 133-148. Springer (2007)

17. Grätzer, G.: General Lattice Theory. Birkhäuser Verlag, 2nd edn. (2003)

18. Hájek, P.: Metamathematics of Fuzzy Logic (Trends in Logic). Springer (2001)

19. Hájek, P.: Making fuzzy description logic more general. Fuzzy Set. Syst. 154(1), $1-15(2005)$ 
20. Horrocks, I., Sattler, U.: A description logic with transitive and inverse roles and role hierarchies. J. Logic and Computation 9(3), 385-410 (1999)

21. Jiang, Y., Tang, Y., Wang, J., Deng, P., Tang, S.: Expressive fuzzy description logics over lattices. Knowl.-Based Syst. 23(2), 150-161 (2010)

22. Molitor, R., Tresp, C.B.: Extending description logics to vague knowledge in medicine. In: Fuzzy Systems in Medicine, Studies in Fuzziness and Soft Computing, vol. 41, pp. 617-635. Springer (2000)

23. Motik, B., Shearer, R., Horrocks, I.: Optimized reasoning in description logics using hypertableaux. In: Proc. CADE'07. LNCS, vol. 4603, pp. 67-83. Springer (2007)

24. Post, E.L.: A variant of a recursively unsolvable problem. Bull. AMS 52(4), 264-268 (1946)

25. Stoilos, G., Straccia, U., Stamou, G., Pan, J.Z.: General concept inclusions in fuzzy description logics. In: Proc. ECAI'06. pp. 457-461. IOS Press (2006)

26. Straccia, U.: Description logics over lattices. Int. J. Uncert. Fuzz. 14(1), 1-16 (2006)

27. Tobies, S.: Complexity Results and Practical Algorithms for Logics in Knowledge Representation. Ph.D. thesis, RWTH Aachen (2001)

28. Viana, H., Alcântara, J., Martins, A.T.C.: Paraconsistent rough description logic. In: Proc. DL'11. CEUR, vol. 745 (2011) 